\title{
CENSO DA ARBORIZAÇÃO DO CAMPUS III - CENTRO POLITÉCNICO DA UNIVERSIDADE FEDERAL DO PARANÁ
}

\section{PLANT CENSUS OF CAMPUS III - CENTRO POLITÉCNICO OF THE UNIVERSIDADE FEDERAL DO PARANÁ}

\author{
Luciana LEAL ${ }^{1}$ \\ José Henrique PEDROSA-MACEDO² \\ Daniela $\mathrm{BIONDI}^{3}$
}

\begin{abstract}
RESUMO
Este trabalho teve como objetivo identificar e caracterizar as plantas encontradas na arborização do Campus III, Centro Politécnico da Universidade Federal do Paraná. No censo realizado foram encontradas 5034 plantas correspondentes a 178 espécies, destas $67,42 \%$ foram árvores, 20,79\% arbustos, 7,86\% plantas herbáceas, 2,24\% palmeiras e 1,68\% pteridófitas. Cerca de $80 \%$ dos indivíduos e $60 \%$ das espécies foram exóticas. As espécies exóticas correspondem a $100 \%$ das plantas herbáceas, $78,38 \%$ dos arbustos, $54,17 \%$ das árvores e $75,00 \%$ das palmeiras. Em relação às espécies nativas, $55,22 \%$ são ocorrentes no ecossistema Floresta Ombrófila Mista e na cidade de Curitiba. Ligustrum lucidum, a espécie arbórea de maior ocorrência, é uma planta invasora. Outras 10 espécies arbóreas ficaram reconhecidas como espécies exóticas invasoras no ecossistema local. Para adequação da arborização do Campus deverão ser realizadas medidas de substituição das espécies invasoras e plantio de espécies nativas.

Palavras-chave: Campus universitário; arborização urbana; plantas nativas; plantas invasoras.
\end{abstract}

\begin{abstract}
These studies had as a goal identify and characterize the plants found in Campus III at the Centro Politécnico of Universidade Federal do Paraná. On the research was found 5034 plants corresponding to 178 species, which $67 \%$ were trees, $21 \%$ shrubs, $8 \%$ herbaceous, $2 \%$ palm trees and $2 \%$ pteridophytes. About $80 \%$ of them and $60 \%$ of the species were exotics. The exotic species correspond to $100 \%$ of the herbaceous, $78 \%$ of the shrubs, $54 \%$ of the trees and $75 \%$ of the palm trees. On native species, $55 \%$ belong to the Floresta Ombrófila Mista ecosystem and to the city of Curitiba. Ligustrum lucidum, the most common tree specie, is an invasive plant. Other 10 tree species were recognized as invaders exotic species at the local ecosystem. In order to adapt the trees of the Campus there are measures that should be followed like invaders species replacement and native species planting.
\end{abstract}

Key-words: University Campus; urban trees; native plants; exotic plants; invasive plants.

1Engenheira Florestal, doutoranda em Engenharia Florestal - Universidade Federal do Paraná (UFPR), Curitiba, Paraná, Brasil. E-mail: luciana_paisagem@yahoo.com.br;

2Engenheiro Florestal, Prof. Dr. Depto. Ciências Florestais - Universidade Federal do Paraná (UFPR), Curitiba, Paraná, Brasil. E-mail: johpema@netpar.com.br;

${ }^{3}$ Engenheira Florestal, Profa. Dra. Depto. Ciências Florestais - Universidade Federal do Paraná (UFPR), pesquisadora bolsista do CNPq. Av. Lothário Meissner, 900, Jardim Botânico, Curitiba - PR, 80.210-170. E-mail: dbiondi@ufpr.br Autora para correspondência. 


\section{INTRODUÇÃO}

Muitas universidades brasileiras possuem em seus Campi Universitários áreas arborizadas com diferentes espécies. Conhecer e catalogar estas espécies pode ter muitas aplicações. Para Eisenlohr et al. (2006), os Campi das grandes universidades brasileiras apresentam, em geral, reconhecido potencial para o desenvolvimento de sólidas pesquisas em arborização urbana e paisagismo. A motivação intelectual inerente ao meio acadêmico, aliada ao espaço urbano peculiar das instituições de ensino superior, tem sido decisiva para a realização, nestes locais, de alguns estudos relevantes naquela área do conhecimento.

No Campus I da Universidade de Passo Fundo (UPF), Melo \& Severo (2007) realizaram pesquisa com o objetivo de catalogar, conservar e divulgar o valor das espécies arbóreas, bem como proporcionar à comunidade local a oportunidade de nova postura perante a vegetação, sensibilizando-a quanto a sua importância, aliada à responsabilidade social da Universidade. Segundo estes autores, a presença da floresta urbana do Campus traz qualidade de vida a seus estudantes, comprometendo-os com a responsabilidade social em relação a sua preservação e conservação.

Paiva (2006) realizou um censo da vegetação da Praça do Relógio da Cidade Universitária "Armando de Salles Oliveira" da Universidade de São Paulo (USP), para incentivar o seu uso como área para estudo de ciências biológicas e educação ambiental, além de fornecer subsídios para a sua manutenção. A autora ainda ressalta a importância de espaços como este na qualidade do ambiente da cidade e na vida dos cidadãos.

Kurihara et al. (2005) realizaram estudo para descrever a quantidade e a qualidade das árvores encontradas no Campus da Universidade de Brasília (UnB), com intuito de valorizar a arborização ali existente. Paiva et al. (2004) realizaram trabalho de levantamento e caracterização das espécies arbóreas localizadas em canteiro central do Campus da Universidade Federal de Lavras (UFLA), para o uso das plantas em pesquisas, coleta de sementes e aulas práticas. Para Parmaksiz et al. (2006), os Campi Universitários podem ser considerados como um laboratório natural que serve como o primeiro material para uso nas disciplinas relacionadas com a botânica.

Nobre \& Menezes (2006) realizaram diagnóstico das espécies que são usadas no paisagismo do Campus Marco Zero do Equador da Universidade Federal do Amapá (UNIFAP), como subsídio para a transformação do Campus em um espaço adequado para a comunidade acadêmica e local, proporcionando uma paisagem propícia ao trabalho e lazer, além de disponibilizar materiais didáticos e de pesquisa.

Faleiro \& Amâncio-Pereira (2007), em estudo no Campus Umuarama da Universidade Federal de Uberlândia (UFU), verificaram uma alta diversidade florística e uma excelente arborização, proporcionando um ambiente agradável à comunidade e um refúgio a invertebrados e à avifauna, pela oferta de alimentos e proteção ambiental.

Independente dos espaços serem em cidades universitárias ou em pátios escolares, a colocação e o conhecimento da vegetação tem a mesma função ou aplicação para seus usuários. Segundo Biondi et al. (2008), a intervenção paisagística nos pátios das escolas deveria ser planejada não apenas para atender às necessidades estéticas e de conforto ambiental dos usuários como, também, para servir de ferramenta às práticas de ensino. A vegetação, matéria-prima do paisagismo, deve ser usada para ilustrar os conhecimentos teóricos relativos à conservação da natureza.

O tratamento paisagístico das áreas externas das unidades de ensino tem como objetivo principal, a melhoria de sua qualidade visual e ambiental (Schaffer, 2005). A composição da vegetação adotada em cada projeto paisagístico deve valorizar e potencializar o uso de áreas externas para atividades pedagógicas e recreativas, além de contribuir para o conforto microclimático dos espaços internos e externos das unidades de ensino (Fedrizzi, 1999).

No Campus III - Centro Politécnico, maior Campus da Universidade Federal do Paraná (UFPR), a arborização é composta por espécies que foram plantadas, tanto pelos técnicos da Prefeitura Universitária, como por professores e estudantes, com diversas finalidades. Visto que não há citações de estudos que avaliem as espécies que compõe a arborização deste Campus, este trabalho teve como objetivo identificar e caracterizar as plantas encontradas.

\section{MATERIAL E MÉTODOS}

Este estudo foi realizado no Campus III, Centro Politécnico da Universidade Federal do Paraná (UFPR), localizado no bairro Jardim das Américas, cidade de Curitiba - PR, situada a $25^{\circ} 25^{\prime}$ de latitude sul e $49^{\circ} 16^{\prime}$ de longitude oeste, com área total aproximada de 64 ha.

Como área de arborização considerou-se as áreas verdes entre os prédios, ruas, estacionamentos e canteiros centrais. Foram desconsiderados os remanescentes florestais presentes no Campus.

No levantamento, realizado no mês de novembro de 2007, foi adotado o método de inventário do tipo censo, com a medição de todas as plantas perenes (incluindo-se mudas).

Indivíduos de todas as espécies estudadas foram herborizadas e identificadas no Herbário do Setor de Ciências Biológicas da Universidade Federal do Paraná e no Museu do Jardim Botânico da Prefeitura Municipal de Curitiba. Os nomes científicos e a autoria dos epítetos específicos foram conferidos pelo banco eletrônico do Jardim Botânico de Missouri (MISSOURI BOTANICAL 
LEAL, L. et al. Censo da arborização do Campus III...

GARDEN, 2008).

Para caracterizar a arborização deste Campus, considerou-se, conforme Michalenko (2002), os seguintes critérios: diversificação de espécies, presença de espécies nativas e não ocorrência de espécies invasoras.

As plantas foram classificadas em quatro grupos de acordo com a sua forma de vida, conforme Biondi (1990):

a) árvore - toda vegetação lenhosa com tronco, copa definida e tamanho superior a $6 \mathrm{~m}$;

b) arbusto - toda vegetação lenhosa, com bifurcação a baixa altura ou rente ao solo, de tamanho inferior a $6 \mathrm{~m}$;

c) herbácea - toda planta com caule não lenhoso ou semi-lenhoso de porte variado, podendo adquirir a altura e os efeitos de um arbusto;

d) palmeira - planta de porte variado com aspecto característico tanto do tronco como da copa.

Além destes grupos, as plantas também tiveram a classificação em pteridófitas que, segundo Ferri (1976), se caracterizam por não produzirem flores. Seu caule, no caso mais freqüente, é um rizoma do qual nascem, de um lado, raízes, de outro, folhas.

As espécies também foram caracterizadas como espécies nativas no Brasil, nativas em Curitiba, exóticas e invasoras, conforme os seguintes critérios: a) espécie nativa no Brasil - ocorre espontaneamente nos ecossistemas existentes em território brasileiro;

b) espécie nativa em Curitiba - ocorre espontaneamente no ecossistema de Floresta Ombrófila Mista (Floresta com Araucária), onde o município de Curitiba está inserido:

c) espécie exótica - não ocorre espontaneamente nos ecossistemas existentes em território brasileiro;

d) espécie invasora - trazida de outros ambientes ou regiões ecológicas, cujas populações são capazes de avançar sobre ecossistemas naturais, causando impactos ambientais e sócioeconômicos. A classificação das espécies invasoras no ecossistema Floresta Ombrófila Mista foi definida a partir da Portaria IAP० o 95 que reconhece a Lista Oficial de Espécies Exóticas Invasoras para o Estado do Paraná (PARANÁ, 2007).

$\mathrm{Na}$ arborização do Campus também foi analisada a presença de espécies frutíferas.

\section{RESULTADOS E DISCUSSÃO}

No Campus III do Centro Politécnico foram encontradas 5034 plantas, correspondentes a 178 espécies, 134 gêneros e 67 famílias (Tabelas 1 a 5). As famílias botânicas com maior número de espécies foram Fabaceae (22 espécies), Cupressaceae (14) e Myrtaceae (12).

TABELA 1 - Árvores encontradas no Campus III - Centro Politécnico - Universidade Federal do Paraná, Curitiba $-2007$

\begin{tabular}{|c|c|c|c|c|c|c|c|c|}
\hline № & NOME CIENTÍFICO & $\begin{array}{c}\text { NOME } \\
\text { VULGAR }\end{array}$ & FAMÍLIA & $P$ & FA & $\%$ & PA & CAR. \\
\hline 1 & Ligustrum lucidum W. T. Aiton & alfeneiro & Oleaceae & $E$ & 724 & 27,17 & 27,17 & $F(A), I$ \\
\hline 2 & Tipuana tipu (Benth.) Kuntze & tipuana & Fabaceae & $\mathrm{N}$ & 166 & 6,23 & 33,40 & \\
\hline 3 & Eucalyptus viminalis Labill. & eucalipto & Myrtaceae & $E$ & 161 & 6,04 & 39,44 & \\
\hline 4 & $\begin{array}{l}\text { Araucaria angustifolia (Bertol.) } \\
\text { Kuntze }\end{array}$ & $\begin{array}{l}\text { pinheiro-do- } \\
\text { paraná }\end{array}$ & Araucariaceae & $\mathrm{NC}$ & 157 & 5,89 & 45,33 & $F(A, H)$ \\
\hline 5 & Lagerstroemia indica L. & extremosa & Lythraceae & $E$ & 131 & 4,92 & 50,24 & \\
\hline 6 & Pinus taeda L. & pinus & Pinaceae & $E$ & 109 & 4,09 & 54,33 & I \\
\hline 7 & Eugenia uniflora L. & pitanga & Myrtaceae & $\mathrm{NC}$ & 81 & 3,04 & 57,37 & $F(A, H)$ \\
\hline 8 & Magnolia grandiflora L. & $\begin{array}{l}\text { magnólia- } \\
\text { branca }\end{array}$ & Magnoliaceae & $E$ & 78 & 2,93 & 60,30 & \\
\hline 9 & $\begin{array}{l}\text { Platanus acerifolia (Aiton) } \\
\text { Willd. }\end{array}$ & plátano & Platanaceae & E & 70 & 2,63 & 62,93 & \\
\hline 10 & Psidium cattleianum Sabine & araçazeiro & Myrtaceae & $\mathrm{N}$ & 58 & 2,18 & 65,10 & $F(A, H)$ \\
\hline 11 & $\begin{array}{l}\text { Viburnum odoratissimum Ker } \\
\text { Gawl. }\end{array}$ & viburno & Caprifoliaceae & $E$ & 57 & 2,14 & 67,24 & \\
\hline 12 & Morus nigra L. & amoreira & Moraceae & $E$ & 56 & 2,10 & 69,34 & $F(A, H), I$ \\
\hline 13 & Liquidambar styraciflua L. & liquidâmbar & Hammamelidaceae & $E$ & 50 & 1,88 & 71,22 & \\
\hline 14 & Jacaranda mimosifolia D. Don & $\begin{array}{l}\text { jacarandá- } \\
\text { mimoso }\end{array}$ & Bignoniaceae & $\mathrm{N}$ & 47 & 1,76 & 72,98 & \\
\hline 15 & Schinus terebinthifolius Raddi & aroeira & Anacardiaceae & $\mathrm{NC}$ & 43 & 1,61 & 74,60 & $F(A)$ \\
\hline 16 & $\begin{array}{l}\text { Tabebuia chrysotricha (Mart ex } \\
\text { A. DC.) Standl. }\end{array}$ & ipê-amarelo & Bignoniaceae & $\mathrm{N}$ & 43 & 1,61 & 76,21 & \\
\hline 17 & $\begin{array}{l}\text { Eriobotrya japonica (Thunb.) } \\
\text { Lindl. }\end{array}$ & nêspera & Rosaceae & E & 35 & 1,31 & 77,52 & $F(A, H), I$ \\
\hline 18 & $\begin{array}{l}\text { Anadenanthera colubrina } \\
\text { (Vell.) Brenan }\end{array}$ & monjoleiro & Fabaceae & $\mathrm{N}$ & 31 & 1,16 & 78,69 & \\
\hline
\end{tabular}


LEAL, L. et al. Censo da arborização do Campus III...

TABELA 1 - Árvores encontradas no Campus III - Centro Politécnico - Universidade Federal do Paraná, Curitiba -2007 (CONTINUAÇÃO)

\begin{tabular}{|c|c|c|c|c|c|c|c|c|}
\hline № & NOME CIENTÍFICO & $\begin{array}{c}\text { NOME } \\
\text { VULGAR }\end{array}$ & FAMÍLIA & $P$ & FA & $\%$ & PA & CAR. \\
\hline 19 & Hovenia dulcis Thunb. & uva-do-japão & Rhamnaceae & $\bar{E}$ & 30 & 1,13 & 79,81 & $F(A, H), I$ \\
\hline 20 & Michelia champaca L. & $\begin{array}{l}\text { magnólia- } \\
\text { amarela }\end{array}$ & Magnoliaceae & $E$ & 30 & 1,13 & 80,94 & $F(A)$ \\
\hline 21 & Cupressus lusitanica Mill. & cipreste & Cupressaceae & E & 26 & 0,98 & 81,91 & \\
\hline 22 & Cassia leptophylla Vog. & $\begin{array}{l}\text { cássia- } \\
\text { fastuosa }\end{array}$ & Fabaceae & $\mathrm{N}$ & 22 & 0,83 & 82,74 & \\
\hline 23 & $\begin{array}{l}\text { Eucalyptus cinerea F. Muell. ex } \\
\text { Benth. }\end{array}$ & eucalipto & Myrtaceae & $E$ & 22 & 0,83 & 83,56 & \\
\hline 24 & Lafoensia pacari A. St.-Hil. & dedaleiro & Lythraceae & NC & 22 & 0,83 & 84,39 & \\
\hline 25 & Butia capitata (Mart.) Becc. & butiá & Arecaceae & $\mathrm{N}$ & 19 & 0,71 & 85,10 & $F(A, H)$ \\
\hline 26 & Psidium guajava Raddi & goiabeira & Myrtaceae & $E$ & 17 & 0,64 & 85,74 & $F(A, H), I$ \\
\hline 27 & Melia azedarach L. & cinamomo & Meliaceae & E & 16 & 0,60 & 86,34 & I \\
\hline 28 & Persea americana Mill. & abacate & Lauraceae & E & 16 & 0,60 & 86,94 & $F(A, H)$ \\
\hline 29 & Cotoneaster francheti Bois & cotoneaster & Rosaceae & E & 13 & 0,49 & 87,43 & \\
\hline 30 & $\begin{array}{l}\text { Cupressus sempervirens L. var. } \\
\text { stricta Aiton }\end{array}$ & cipreste & Cupressaceae & $\mathrm{E}$ & 13 & 0,49 & 87,92 & \\
\hline 31 & Pinus insignis Douglas ex Lould & pinus & Pinaceae & E & 13 & 0,49 & 88,41 & \\
\hline 32 & $\begin{array}{l}\text { Tabebuia heptaphylla (Vell.) } \\
\text { Toledo }\end{array}$ & Ipê-roxo & Bignoniaceae & N & 13 & 0,49 & 88,89 & \\
\hline 33 & Myrsine coriaceae (Sw.) R. Br. & capororoca & Myrsinaceae & $\mathrm{N}$ & 12 & 0,45 & 89,34 & \\
\hline 34 & $\begin{array}{l}\text { Campomanesia xanthocarpa } 0 . \\
\text { Berg. }\end{array}$ & guabirova & Myrtaceae & NC & 11 & 0,41 & 89,76 & $F(A, H)$ \\
\hline 35 & Citrus $\mathrm{x}$ limon L. Burmann & limoeiro & Rutaceae & E & 11 & 0,41 & 90,17 & $F(A, H), I$ \\
\hline 36 & $\begin{array}{l}\text { Allophylus edulis (A. St.-Hil., } \\
\text { Cambess \& A. Juss.) Radlk }\end{array}$ & vacum & Sapindaceae & NC & 10 & 0,38 & 90,54 & $F(A)$ \\
\hline 37 & Prunus persica (L.) Batsch & pessegueiro & Rosaceae & $E$ & 10 & 0,38 & 90,92 & $F(A, H)$ \\
\hline 38 & $\begin{array}{l}\text { Podocarpus lambertii Klotzsch } \\
\text { ex Endl. }\end{array}$ & pinheiro-bravo & Podocarpaceae & NC & 9 & 0,34 & 91,26 & \\
\hline 39 & $\begin{array}{l}\text { Populus deltoides Bartr. ex } \\
\text { Marshall }\end{array}$ & pópulus & Salicaceae & $E$ & 9 & 0,34 & 91,59 & \\
\hline 40 & Tabebuia alba (Cham.) Sandwith & ipê-amarelo & Bignoniaceae & NC & 9 & 0,34 & 91,93 & S \\
\hline 41 & Acer palmatum Thunb. & acer-palmato & Aceraceae & $E$ & 8 & 0,30 & 92,23 & \\
\hline 42 & Ocotea puberula (Rich.) Nees & canela-guaicá & Lauraceae & NC & 8 & 0,30 & 92,53 & $F(A)$ \\
\hline 43 & $\begin{array}{l}\text { Chamaecyparis obtusa (Siebold } \\
\text { \& Zucc.) Endl. "Crippsii" }\end{array}$ & $\begin{array}{l}\text { cipreste- } \\
\text { dourado }\end{array}$ & Cupressaceae & $E$ & 7 & 0,26 & 92,80 & \\
\hline 44 & Chorisia speciosa A. St.-Hil. & paineira & Bombacaceae & $\mathrm{N}$ & 7 & 0,26 & 93,06 & $F(A)$ \\
\hline 45 & Cupressus macrocarpha Hartw. & cedro & Cupressaceae & $E$ & 7 & 0,26 & 93,32 & \\
\hline 46 & $\begin{array}{l}\text { Casuarina equisetifolia J. R. \& } \\
\text { G. Forst. }\end{array}$ & casuarina & Casuarinaceae & $E$ & 6 & 0,23 & 93,55 & \\
\hline 47 & Cupressus macrocarpa Hartw. & $\begin{array}{l}\text { cupresso- } \\
\text { morterey }\end{array}$ & Cupressaceae & $E$ & 6 & 0,23 & 93,77 & \\
\hline 48 & Cydonia oblonga Mill. & marmeleiro & Rosaceae & E & 6 & 0,23 & 94,00 & $F(A, H)$ \\
\hline 49 & Erythrina speciosa Andr. & corticeira & Fabaceae & $\mathrm{N}$ & 6 & 0,23 & 94,22 & \\
\hline 50 & Pittosporum undulatum Vent. & pau-incenso & Pittosporaceae & E & 6 & 0,23 & 94,45 & $F(A), I$ \\
\hline 51 & $\begin{array}{l}\text { Chamaecyparis pisifera Sieb. \& } \\
\text { Zucc. var. filifera-aurea Beissn. }\end{array}$ & tuia-macarrão & Cupressaceae & $E$ & 5 & 0,19 & 94,63 & \\
\hline 52 & Juniperus chinensis L. & junípero-chinês & Cupressaceae & E & 5 & 0,19 & 94,82 & \\
\hline 53 & Thuja occidentalis L. & tuia-maçã & Cupressaceae & $E$ & 5 & 0,19 & 95,01 & \\
\hline 54 & $\begin{array}{l}\text { Parapiptadenia rigida (Benth) } \\
\text { Brenan }\end{array}$ & angico & Fabaceae & N & 5 & 0,19 & 95,20 & \\
\hline 55 & Populus nigra L. & álamo & Salicaceae & E & 5 & 0,19 & 95,38 & \\
\hline 56 & Robinia pseudoacacia L. & acácia-falsa & Fabaceae & E & 5 & 0,19 & 95,57 & I \\
\hline 57 & Vitex montevidensis Cham. & tarumã & Verbenaceae & NC & 5 & 0,19 & 95,76 & $F(A)$ \\
\hline 58 & $\begin{array}{l}\text { Cunninghamia lanceolata } \\
\text { (Lamb.) Hooker }\end{array}$ & pinheiro-chinês & Taxodiaceae & $E$ & 4 & 0,15 & 95,91 & \\
\hline 59 & Cryptomeria japonica D. Don & $\begin{array}{l}\text { pinheiro-do- } \\
\text { japão }\end{array}$ & Taxodiaceae & $E$ & 4 & 0,15 & 96,06 & \\
\hline
\end{tabular}


LEAL, L. et al. Censo da arborização do Campus III...

TABELA 1 - Árvores encontradas no Campus III - Centro Politécnico - Universidade Federal do Paraná, Curitiba - 2007 (CONTINUAÇÃO)

\begin{tabular}{|c|c|c|c|c|c|c|c|c|}
\hline № & NOME CIENTÍFICO & $\begin{array}{c}\text { NOME } \\
\text { VULGAR } \\
\end{array}$ & FAMÍLIA & $\mathrm{P}$ & FA & $\%$ & PA & CAR. \\
\hline 60 & Ficus benjamina L. & $\begin{array}{l}\text { figueira- } \\
\text { benjamina }\end{array}$ & Moraceae & $E$ & 4 & 0,15 & 96,21 & \\
\hline 61 & $\begin{array}{l}\text { Juniperus chinensis L. var } \\
\text { pfitzeriana Spaet. }\end{array}$ & junípero-chinês & Cupressaceae & $E$ & 4 & 0,15 & 96,36 & \\
\hline 62 & $\begin{array}{l}\text { Senna macranthera (DC. } \\
\text { ex Collad) H. S. Irwin \& } \\
\text { Barneby } \\
\text { Chamaecyparis obtusa }\end{array}$ & chuva-de-ouro & Fabaceae & $\mathrm{N}$ & 4 & 0,15 & 96,51 & $F(A)$ \\
\hline 63 & $\begin{array}{l}\text { Siebold \& Zucc. var. } \\
\text { nana-gracilis Beissner }\end{array}$ & cipreste & Cupressaceae & $E$ & 3 & 0,11 & 96,62 & \\
\hline 64 & Cedrela fissilis Vellozo & cedro-rosa & Meliaceae & NC & 3 & 0,11 & 96,74 & \\
\hline 65 & Diospyros kaki L. & caqui & Ebenaceae & $E$ & 3 & 0,11 & 96,85 & $F(A, H)$ \\
\hline 66 & $\begin{array}{l}\text { Ilex paraguariensis A. St.- } \\
\text { Hil. }\end{array}$ & erva-mate & Aquifoliaceae & $\mathrm{NC}$ & 3 & 0,11 & 96,96 & $F(A)$ \\
\hline 67 & Liriodendron tulipifera L. & liriodendro & Magnoliaceae & $E$ & 3 & 0,11 & 97,07 & \\
\hline 68 & Pyrus communis L. & pereira & Rosaceae & $E$ & 3 & 0,11 & 97,19 & $F(A, H)$ \\
\hline 69 & Senna sp & sena & Fabaceae & $\mathrm{N}$ & 3 & 0,11 & 97,30 & \\
\hline 70 & $\begin{array}{l}\text { Solanum pseudoquina A. } \\
\text { St.-Hil. }\end{array}$ & quina & Solanaceae & $\mathrm{NC}$ & 3 & 0,11 & 97,41 & \\
\hline 71 & $\begin{array}{l}\text { Taxodium distichum (L.) } \\
\text { Rich. }\end{array}$ & $\begin{array}{l}\text { pinheiro-das- } \\
\text { folhas- } \\
\text { pêndulas }\end{array}$ & Taxodiaceae & $E$ & 3 & 0,11 & 97,52 & \\
\hline 72 & $\begin{array}{l}\text { Tibouchina sellowiana } \\
\text { Cogn. } \\
\text { Chamaecyparis }\end{array}$ & quaresmeira & Melastomataceae & $\mathrm{NC}$ & 3 & 0,11 & 97,64 & \\
\hline 73 & $\begin{array}{l}\text { lawsoniana (A. Mun.) } \\
\text { Parl. "Allumii" }\end{array}$ & cipreste-alumi & Cupressaceae & $E$ & 2 & 0,08 & 97,71 & \\
\hline 74 & $\begin{array}{l}\text { Chamaecyparis pisifera } \\
\text { Sieb. \& Zucc. "Boulevard" }\end{array}$ & tuia-azul & Cupressaceae & $E$ & 2 & 0,08 & 97,79 & \\
\hline 75 & $\begin{array}{l}\text { Juniperus chinensis L. } \\
\text { "Torulosa" }\end{array}$ & junípero-chinês & Cupressaceae & $E$ & 2 & 0,08 & 97,86 & \\
\hline 76 & $\begin{array}{l}\text { Cedrela odorata L. } \\
\text { Enterolobium }\end{array}$ & cedro-cheiroso & Meliaceae & $\mathrm{N}$ & 2 & 0,08 & 97,94 & \\
\hline 77 & $\begin{array}{l}\text { contortisiliquum (Vell.) } \\
\text { Morong }\end{array}$ & timbaúva & Fabaceae & $\mathrm{N}$ & 2 & 0,08 & 98,01 & \\
\hline 78 & Eucalyptus dunni Maiden & eucalipto & Myrtaceae & $E$ & 2 & 0,08 & 98,09 & \\
\hline 79 & Eugenia involucrata DC. & cerejeira & Myrtaceae & $\mathrm{NC}$ & 2 & 0,08 & 98,16 & $F(A, H)$ \\
\hline 80 & Ficus carica L. & figueira & Moraceae & $E$ & 2 & 0,08 & 98,24 & $F(A, H)$ \\
\hline 81 & $\begin{array}{l}\text { Leucaena leucocephala } \\
\text { (Lam.) R. de Wit. }\end{array}$ & leucena & Fabaceae & $E$ & 2 & 0,08 & 98,31 & I \\
\hline 82 & Mangifera indica L. & mangueira & Anacardiaceae & $E$ & 2 & 0,08 & 98,39 & $F(A, H)$ \\
\hline 83 & $\begin{array}{l}\text { Rollinea sericia (R. E. } \\
\text { Fries) R. E. Fries }\end{array}$ & ariticum & Annonaceae & $\mathrm{N}$ & 2 & 0,08 & 98,46 & $F(A, H)$ \\
\hline 84 & Salix babilonica L. & chorão & Salicaceae & $E$ & 2 & 0,08 & 98,54 & \\
\hline 85 & $\begin{array}{l}\text { Sebastiania brasiliensis } \\
\text { Spreng. } \\
\text { Sebastiania }\end{array}$ & $\begin{array}{l}\text { branquilho- } \\
\text { graúdo }\end{array}$ & Euphorbiaceae & $\mathrm{NC}$ & 2 & 0,08 & 98,61 & $F(A)$ \\
\hline 86 & $\begin{array}{l}\text { commersoniana (Bailon) } \\
\text { L. B. Smith \& R. J. Downs }\end{array}$ & branquilho & Euphorbiaceae & $\mathrm{NC}$ & 2 & 0,08 & 98,69 & $F(A)$ \\
\hline 87 & Symplocos sp & maria-mole & Symplocaceae & $\mathrm{NC}$ & 2 & 0,08 & 98,76 & $F(A)$ \\
\hline 88 & Acacia mearnsii De Wild. & acácia-negra & Fabaceae & $E$ & 1 & 0,04 & 98,80 & 1 \\
\hline 89 & Acacia trinervis Desv. & acácia-trinervis & Fabaceae & $E$ & 1 & 0,04 & 98,84 & \\
\hline 90 & Acer negundo L. & acer & Aceraceae & $E$ & 1 & 0,04 & 98,87 & \\
\hline 91 & Bauhinia forficata Link & pata-de-vaca & Fabaceae & $\mathrm{NC}$ & 1 & 0,04 & 98,91 & \\
\hline 92 & Bixa orellana L. & urucum & Bixaceae & $\mathrm{N}$ & 1 & 0,04 & 98,95 & \\
\hline 93 & $\begin{array}{l}\text { Caesalpinia echinata } \\
\text { Lam. }\end{array}$ & pau-brasil & Fabaceae & $\mathrm{N}$ & 1 & 0,04 & 98,99 & $S$ \\
\hline 94 & $\begin{array}{l}\text { Caesalpinia } \\
\text { peltophoroides Benth. }\end{array}$ & sibipiruna & Fabaceae & $\mathrm{N}$ & 1 & 0,04 & 99,02 & \\
\hline
\end{tabular}


LEAL, L. et al. Censo da arborização do Campus III...

TABELA 1 - Árvores encontradas no Campus III - Centro Politécnico - Universidade Federal do Paraná, Curitiba -2007 (CONTINUAÇÃO)

\begin{tabular}{|c|c|c|c|c|c|c|c|c|}
\hline № & NOME CIENTÍFICO & $\begin{array}{c}\text { NOME } \\
\text { VULGAR }\end{array}$ & FAMÍLIA & $P$ & FA & $\%$ & PA & CAR. \\
\hline 95 & Carica papaya L. & mamoeiro & Caricaceae & $\mathrm{E}$ & 1 & 0,04 & 99,06 & $F(A, H)$ \\
\hline 96 & Casearia sp & guaçatonga & Flacourtiaceae & NC & 1 & 0,04 & 99,10 & $F(A)$ \\
\hline 97 & Casearia sylvestris Sw. & $\begin{array}{l}\text { cafezinho- } \\
\text { bravo }\end{array}$ & Flacourtiaceae & NC & 1 & 0,04 & 99,14 & $F(A)$ \\
\hline 98 & $\begin{array}{l}\text { Chrysophyllum gonocarpum } \\
\text { (Mart. \& Eichler) Eng. }\end{array}$ & guatambu & Sapotaceae & $\mathrm{N}$ & 1 & 0,04 & 99,17 & $F(A)$ \\
\hline 99 & $\begin{array}{l}\text { Citronella gongonha (Mart.) R. } \\
\text { A. Howard }\end{array}$ & congonha & Icacinaceae & NC & 1 & 0,04 & 99,21 & \\
\hline 100 & Cupressus sp & cedro & Cupressaceae & $E$ & 1 & 0,04 & 99,25 & \\
\hline 101 & $\begin{array}{l}\text { Cryptomeria japonica D. Don } \\
\text { "Plumosa" }\end{array}$ & $\begin{array}{l}\text { pinheiro-do- } \\
\text { japão }\end{array}$ & Taxodiaceae & E & 1 & 0,04 & 99,29 & \\
\hline 102 & Enterolobium maximum Ducke & tamboril & Fabaceae & N & 1 & 0,04 & 99,32 & \\
\hline 103 & Erythrina falcata Benth. & corticeira & Fabaceae & NC & 1 & 0,04 & 99,36 & \\
\hline 104 & Eucalyptus citriodora Hook. & eucalipto & Myrtaceae & $E$ & 1 & 0,04 & 99,40 & \\
\hline 105 & $\begin{array}{l}\text { Gochnatia polymorfa subsp. } \\
\text { floccosa (Less.) Cabr. }\end{array}$ & cambará & Asteraceae & NC & 1 & 0,04 & 99,44 & \\
\hline 106 & Inga sessilis (Vell.) Mart. & ingá-macaco & Fabaceae & N & 1 & 0,04 & 99,47 & $F(A, H)$ \\
\hline 107 & Lithraea brasiliensis March. & $\begin{array}{l}\text { pau-de- } \\
\text { bugre }\end{array}$ & Anacardiaceae & NC & 1 & 0,04 & 99,51 & \\
\hline 108 & Luhea divaricata Mart. & $\begin{array}{l}\text { açoita- } \\
\text { cavalo }\end{array}$ & Tiliaceae & $\mathrm{NC}$ & 1 & 0,04 & 99,55 & \\
\hline 109 & Matayba elaeagnoides Radlk. & $\begin{array}{l}\text { miguel- } \\
\text { pintado }\end{array}$ & Sapindaceae & NC & 1 & 0,04 & 99,59 & \\
\hline 110 & Melaleuca leucadendron (L.) L. & melaleuca & Myrtaceae & $E$ & 1 & 0,04 & 99,62 & \\
\hline 111 & Myrcia laruotteana Cambess. & cambuim & Myrtaceae & NC & 1 & 0,04 & 99,66 & \\
\hline 112 & $\begin{array}{l}\text { Peltophorum dubium (Spreng.) } \\
\text { Taub. }\end{array}$ & canafístula & Fabaceae & $\mathrm{N}$ & 1 & 0,04 & 99,70 & \\
\hline 113 & Pinus elliottii Engelm. & pinus & Pinaceae & $E$ & 1 & 0,04 & 99,74 & I \\
\hline 114 & Pinus patula Schltdl. \& Cham. & pinus & Pinaceae & $E$ & 1 & 0,04 & 99,77 & \\
\hline 115 & Prunus sellowii Koehne & $\begin{array}{l}\text { pessegueiro- } \\
\text { bravo }\end{array}$ & Rosaceae & NC & 1 & 0,04 & 99,81 & \\
\hline 116 & Punica granatum $\mathrm{L}$. & romã & Punicaceae & $E$ & 1 & 0,04 & 99,85 & $F(A, H)$ \\
\hline 117 & Quercus robur $\mathrm{L}$. & carvalho & Fagaceae & $E$ & 1 & 0,04 & 99,89 & \\
\hline 118 & $\begin{array}{l}\text { Rapanea ferruginea (Ruiz et } \\
\text { Pav.) Mez }\end{array}$ & $\begin{array}{l}\text { canela } \\
\text { capororoca }\end{array}$ & Myrsinaceae & $\mathrm{N}$ & 1 & 0,04 & 99,92 & \\
\hline 119 & Schinus molle L. & aroeira-salsa & Anacardiaceae & $E$ & 1 & 0,04 & 99,96 & $F(A)$ \\
\hline 120 & Solanum sp & $x$ & Solanaceae & $\mathrm{NC}$ & 1 & 0,04 & 100,00 & \\
\hline
\end{tabular}

LEGENDA: $\mathrm{P}$. = procedência: $\mathrm{N}$ = nativa no Brasil, $\mathrm{NC}$ = nativa em Curitiba; $\mathrm{E}=$ exótica no Brasil, $\mathrm{FA}$ = freqüência absoluta (número de indivíduos), $\mathrm{FR}=$ freqüência relativa $(\%), \mathrm{PA}=$ percentagem acumulada de espécies $(\%), \mathrm{CAR}$. = caracterização das espécies, $F(A)=$ frutífera para avifauna, $F(H)=$ frutífera para humanos, $I=$ planta invasora na Floresta Ombrófila Mista. A caracterização das espécies foi de acordo com Lorenzi (1992), Lorenzi (1998), Lorenzi et al. (2003) e PARANÁ (2007).

TABELA 2 - Arbustos encontrados no Campus III - Centro Politécnico - Universidade Federal do Paraná, Curitiba -2007

\begin{tabular}{cllllllll}
\hline № & \multicolumn{1}{c}{ NOME CIENTíFICO } & $\begin{array}{c}\text { NOME } \\
\text { VULGAR }\end{array}$ & FAMÍLIA & P. & TOTAL & $\%$ & PA & CAR. \\
\hline 1 & Rhododendron simsii Planch. & azaléia & Ericaceae & $\mathrm{E}$ & 143 & 18,48 & 18,48 \\
2 & Jasminum mesnyi Hance & jasmim- & Omarelo & Oleaceae & $\mathrm{E}$ & 103 & 13,31 & 31,78 \\
3 & $\begin{array}{l}\text { Abelia } \text { x grandiflora (Rovelli ex } \\
\text { André) Rehder }\end{array}$ & abélia & Caprifoliaceae & $\mathrm{E}$ & 62 & 8,01 & 39,79 \\
4 & $\begin{array}{l}\text { Hydrangea macrophylla (Thunb.) } \\
\text { Ser. }\end{array}$ & hortênsia & Hydrangeaceae & $\mathrm{E}$ & 58 & 7,49 & 47,29 \\
5 & Hibiscus rosa-sinensis L. & hibisco & Malvaceae & $\mathrm{E}$ & 46 & 5,94 & 53,23 \\
6 & Yucca filamentosa L. & iuca & Liliaceae & $\mathrm{E}$ & 37 & 4,78 & 58,01 \\
\hline
\end{tabular}


LEAL, L. et al. Censo da arborização do Campus III...

TABELA 2 - Arbustos encontrados no Campus III - Centro Politécnico - Universidade Federal do Paraná, Curitiba - 2007 (CONTINUAÇÃO)

\begin{tabular}{|c|c|c|c|c|c|c|c|c|}
\hline № & NOME CIENTÍFICO & NOME VULGAR & FAMÍLIA & P. & TOTAL & $\%$ & PA & CAR. \\
\hline 7 & $\begin{array}{l}\text { Euphorbia milii Des. } \\
\text { Moul. }\end{array}$ & coroa-de-cristo & Euphorbiaceae & $E$ & 34 & 4,39 & 62,40 & \\
\hline 8 & $\begin{array}{l}\text { Duranta repens L. } \\
\text { "Aurea" }\end{array}$ & pingo-de-ouro & Verbenaceae & $E$ & 30 & 3,88 & 66,28 & \\
\hline 9 & Nerium oleander $\mathrm{L}$. & espirradeira & Apocynaceae & $E$ & 29 & 3,75 & 70,03 & \\
\hline 10 & $\begin{array}{l}\text { Cordyline dracaenoides } \\
\text { Kunth }\end{array}$ & uvarana & Liliaceae & NC & 26 & 3,36 & 73,39 & $F(A)$ \\
\hline 11 & $\begin{array}{l}\text { Odontonema strictum } \\
\text { Kuntze }\end{array}$ & odontonema & Acanthaceae & E & 26 & 3,36 & 76,74 & \\
\hline 12 & $\begin{array}{l}\text { Cordyline terminalis } \\
\text { Kunth }\end{array}$ & cordiline & Liliaceae & $E$ & 25 & 3,23 & 79,97 & \\
\hline 13 & Spiraea wilsoni Duthie & grinalda-de-noiva & Rosaceae & $\mathrm{E}$ & 22 & 2,84 & 82,82 & \\
\hline 14 & $\begin{array}{l}\text { Euphorbia pulcherrima } \\
\text { Willd. ex Klotzsch }\end{array}$ & bico-de-papagaio & Euphorbiaceae & $E$ & 21 & 2,71 & 85,53 & \\
\hline 15 & $\begin{array}{l}\text { Caliandra brevipes } \\
\text { Benth. }\end{array}$ & esponjinha & Fabaceae & $\mathrm{N}$ & 15 & 1,94 & 87,47 & \\
\hline 16 & Musa sp & bananeira & Musaceae & $E$ & 14 & 1,81 & 89,28 & $F(A, H))$ \\
\hline 17 & $\begin{array}{l}\text { Tibouchina grandiflora } \\
\text { Cogn. }\end{array}$ & orelha-de-onça & Melastomataceae & E & 11 & 1,42 & 90,70 & \\
\hline 18 & $\begin{array}{l}\text { Tibouchina fothergillae } \\
\text { Cogn. }\end{array}$ & quaresmeira & Melastomataceae & $\mathrm{N}$ & 10 & 1,29 & 91,99 & \\
\hline 19 & Rosa x grandiflora Hort. & roseira & Rosaceae & $E$ & 6 & 0,78 & 92,76 & \\
\hline 20 & Caliandra tweedi Benth. & caliandra & Fabaceae & $\mathrm{N}$ & 5 & 0,65 & 93,41 & \\
\hline 21 & $\begin{array}{l}\text { Callistemon viminalis } \\
\text { (Sol. ex Gaertn.) G. Don } \\
\text { ex Loud. }\end{array}$ & $\begin{array}{l}\text { escova-pendente- } \\
\text { de-garrafa }\end{array}$ & Myrtaceae & E & 5 & 0,65 & 94,06 & \\
\hline 22 & $\begin{array}{l}\text { Malvaviscus arboreus } \\
\text { Cav. }\end{array}$ & malvavisco & Malvaceae & E & 5 & 0,65 & 94,70 & \\
\hline 23 & $\begin{array}{l}\text { Spartium junceum L. } \\
\text { Abutilon }\end{array}$ & giesta & Fabaceae & E & 5 & 0,65 & 95,35 & \\
\hline 24 & $\begin{array}{l}\text { megapotamicum St.-Hil } \\
\& \text { Naudin }\end{array}$ & lanterna-chinesa & Malvaceae & $E$ & 4 & 0,52 & 95,87 & \\
\hline 25 & $\begin{array}{l}\text { Bougainvillea spectabilis } \\
\text { Willd. }\end{array}$ & três-marias & Nyctaginaceae & E & 4 & 0,52 & 96,38 & \\
\hline 26 & Abutilon darwinii Hook & sino-amarelo & Malvaceae & $E$ & 3 & 0,39 & 96,77 & \\
\hline 27 & $\begin{array}{l}\text { Bambusa gracilis Hort. } \\
\text { ex C. Riviere }\end{array}$ & $\begin{array}{l}\text { bambuzinho-de- } \\
\text { jardim }\end{array}$ & Poaceae & E & 3 & 0,39 & 97,16 & \\
\hline 28 & Coffea arabica L. & café & Rubiaceae & $E$ & 3 & 0,39 & 97,55 & \\
\hline 29 & Hibiscus syriacus Linn. & hibisco & Malvaceae & $E$ & 3 & 0,39 & 97,93 & \\
\hline 30 & Petrea subserrata Cham. & viuvinha & Verbenaceae & $E$ & 3 & 0,39 & 98,32 & \\
\hline 31 & $\begin{array}{l}\text { Schefflera arboricola } \\
\text { Hayata }\end{array}$ & cheflera & Araliaceae & E & 3 & 0,39 & 98,71 & \\
\hline 32 & $\begin{array}{l}\text { Tetrapanax papyriferus } \\
\text { K. Koch }\end{array}$ & papel-arroz & Araliaceae & E & 3 & 0,39 & 99,10 & \\
\hline 33 & Bambusa sp & taquara & Poaceae & $\mathrm{N}$ & 2 & 0,26 & 99,35 & \\
\hline 34 & $\begin{array}{l}\text { Gardenia jasminoides J. } \\
\text { Ellis }\end{array}$ & gardênia & Rubiaceae & $E$ & 2 & 0,26 & 99,61 & \\
\hline 35 & $\begin{array}{l}\text { Bougainvillea glabra } \\
\text { Choisy }\end{array}$ & três-marias & Nyctaginaceae & $\mathrm{N}$ & 1 & 0,13 & 99,74 & \\
\hline 36 & Duranta vestita Cham. & esporão-de-galo & Verbenaceae & NC & 1 & 0,13 & 99,87 & \\
\hline \multirow[t]{2}{*}{37} & $\begin{array}{l}\text { Maytenus ilicifolia Mart. } \\
\text { ex Reiss. }\end{array}$ & espinheira-santa & Celastraceae & NC & 1 & 0,13 & 100,00 & \\
\hline & & & & TOTAL & 774 & 100,00 & 100,00 & \\
\hline
\end{tabular}

LEGENDA: $\mathrm{P}$. = procedência: $\mathrm{N}$ = nativa no Brasil, $\mathrm{NC}$ = nativa em Curitiba; $\mathrm{E}=$ exótica no Brasil, $\mathrm{FA}$ = freqüência absoluta (número de indivíduos), $\mathrm{FR}=$ freqüência relativa $(\%), \mathrm{PA}=$ percentagem acumulada de espécies $(\%), \mathrm{CAR} .=$ caracterização das espécies, $F(A)$ = frutífera para avifauna, $F(H)=$ frutífera para humanos

A caracterização das espécies foi de acordo com Lorenzi \& Souza (2001). 
LEAL, L. et al. Censo da arborização do Campus III...

TABELA 3 - Plantas herbáceas perenes encontradas no Campus III - Centro Politécnico - Universidade Federal do Paraná, Curitiba - 2007

\begin{tabular}{cllllllll}
\hline № & \multicolumn{1}{c}{ NOME CIENTíFICO } & NOME VULGAR & FAMÍLIA & P. & TOTAL & $\%$ & PA & CAR. \\
\hline 1 & Polianthus tuberosa Linn & angélica & Amaryllidaceae & $\mathrm{E}$ & 700 & 46,98 & 46,98 \\
2 & Hemerocallis flava (L.) L. & hemerocalis & Liliaceae & $\mathrm{E}$ & 300 & 20,13 & 67,11 \\
3 & Dietes iridioides Sweet & moréia & Iridaceae & $\mathrm{E}$ & 210 & 14,09 & 81,21 \\
4 & Curculigo capitulata Kuntze & capim-palmeira & Amaryllidaceae & $\mathrm{E}$ & 150 & 10,07 & 91,28 \\
5 & Agapanthus africanus (L.) & agapanto & Amaryllidaceae & $\mathrm{E}$ & 50 & 3,36 & 94,63 \\
6 & Hoffmanns & agave americana L. & gazânia & Amaryllidaceae & $\mathrm{E}$ & 32 & 2,15 & 96,78 \\
7 & Gazania rigens Moench & costela-de-adão & Araceae & $\mathrm{E}$ & 6 & 0,40 & 99,19 \\
8 & Monstera deliciosa Liebm. & boldo & Lamiaceae & $\mathrm{E}$ & 3 & 0,20 & 99,40 \\
9 & Plectranthus barbatus Andrews & flor-ave-do-paraíso & Musaceae & $\mathrm{E}$ & 3 & 0,20 & 99,60 \\
10 & Strelitzia reginae Banks & agave & Amaryllidaceae & $\mathrm{E}$ & 2 & 0,13 & 99,73 \\
11 & Agave sp & biri & Cannaceae & $\mathrm{E}$ & 2 & 0,13 & 99,87 \\
12 & Canna indica L. & tomate-japonês & Solanaceae & $\mathrm{E}$ & 1 & 0,07 & 99,93 & F(A, H) \\
13 & Cyphomandra betacea (Cav.) & Sendtn. & Liliaceae & $\mathrm{E}$ & 1 & 0,07 & 100,00 \\
14 & Kniphofia uvaria Hook. & & TOTAL & 1490 & 100,00 & 100,00
\end{tabular}

LEGENDA: $\mathrm{P}$. = procedência: $\mathrm{N}$ = nativa no Brasil, $\mathrm{NC}$ = nativa em Curitiba; $\mathrm{E}$ = exótica no Brasil, $\mathrm{FA}$ = freqüência absoluta (número de indivíduos), $\mathrm{FR}=$ freqüência relativa $(\%), \mathrm{PA}=$ percentagem acumulada de espécies $(\%), \mathrm{CAR} .=$ caracterização das espécies, $F(A)$ = frutífera para avifauna, $F(H)=$ frutífera para humanos.

A caracterização das espécies foi de acordo com Lorenzi \& Souza (2001).

TABELA 4 - Palmeiras encontradas no Campus III - Centro Politécnico - Universidade Federal do Paraná, Curitiba - 2007

\begin{tabular}{|c|c|c|c|c|c|c|c|c|}
\hline № & NOME CIENTÍFICO & NOME VULGAR & FAMÍLIA & $\mathrm{P}$. & TOTAL & $\%$ & PA & CAR. \\
\hline 1 & $\begin{array}{l}\text { Syagrus romanzoffiana (Cham.) } \\
\text { Glassm. }\end{array}$ & jerivá & Arecaceae & $\mathrm{NC}$ & 85 & 91,40 & 91,40 & $F(A)$ \\
\hline 2 & Phoenix roebelinii O' Brien & $\begin{array}{l}\text { tamareira-de- } \\
\text { jardim }\end{array}$ & Arecaceae & $E$ & 4 & 4,30 & 95,70 & \\
\hline 3 & Chamaerops humilis L. & $\begin{array}{l}\text { palmeira-moinho- } \\
\text { de-vento }\end{array}$ & Arecaceae & E & 2 & 2,15 & 97,84 & \\
\hline \multirow[t]{2}{*}{4} & $\begin{array}{l}\text { Washingtonia filifera (Linden) } \\
\text { Wendland }\end{array}$ & washigtonia & Arecaceae & $E$ & 2 & 2,15 & 100,00 & \\
\hline & & & & TAL & 93 & 100,00 & 100,00 & \\
\hline
\end{tabular}

LEGENDA: $\mathrm{P}$. = procedência: $\mathrm{N}$ = nativa no Brasil, $\mathrm{NC}$ = nativa em Curitiba; $\mathrm{E}=$ exótica no Brasil, $\mathrm{FA}$ = freqüência absoluta (número de indivíduos), $\mathrm{FR}=$ freqüência relativa $(\%), \mathrm{PA}=$ percentagem acumulada de espécies $(\%), \mathrm{CAR} .=$ caracterização das espécies, $F(A)=$ frutífera para avifauna, $F(H)=$ frutífera para humanos.

A caracterização das espécies foi de acordo com Lorenzi \& Souza (2001).

TABELA 5 - Pteridófitas encontradas no Campus III - Centro Politécnico - Universidade Federal do Paraná, Curitiba - 2007

\begin{tabular}{cllllccc}
\hline № & \multicolumn{1}{c}{ NOME CIENTíFICO } & \multicolumn{1}{c}{ NOME VULGAR } & FAMÍLIA & P. & TOTAL & $\%$ & PA \\
\hline 1 & Dicksonia sellowiana Hook. & xaxim & Dicksoniaceae & NC & 5 & 41,67 & 41,67 \\
2 & Blechnum brasiliense Desv. & samambaiaçu-do-brejo & Polypodiaceae & NC & 5 & 41,67 & 83,34 \\
3 & Nephelea setosa (Kaulf.) Tryon & xaxim-arborescente & Ciataceae & NC & 2 & 16,67 & 100,00 \\
\hline & & & & TOTAL & 12 & 100,00 & 100,00 \\
\hline
\end{tabular}

LEGENDA: $\mathrm{P}$. = procedência: $\mathrm{N}$ = nativa no Brasil, $\mathrm{NC}$ = nativa em Curitiba; $\mathrm{E}$ = exótica no Brasil, $\mathrm{FA}$ = freqüência absoluta (número de indivíduos), $\mathrm{FR}$ = freqüência relativa (\%), PA = percentagem acumulada de espécies (\%).

Do total de espécies, $120(67,42 \%)$ são árvores (Tabela 1), $37(20,79 \%)$ são arbustos (Tabela 2), 14 (7,86\%) são plantas herbáceas perenes (Tabela 3$)$, quatro $(2,24 \%)$ são palmeiras (Tabela 4$)$ e três $(1,68 \%)$ são pteridófitas (Tabela 5). Levando em consideração o espaço educativo em que se encontram, estas espécies podem ser utilizadas como material didático em aulas práticas e como plantas matrizes para propagação, conforme expuseram Paiva et al. (2004), Kurihara et al. (2005) e Nobre \& Menezes (2006). Também podem proporcionar educação ambiental aos que visitam o Campus e aos que passam por ele diariamente. 
LEAL, L. et al. Censo da arborização do Campus III...

O maior número das espécies e dos indivíduos encontrados, 120 e 2665, respectivamente (Tabela 1), corresponde às árvores. Ao se analisar a relação do número de plantas por espécie, verificou-se que as cinco espécies arbóreas de maior ocorrência correspondem a mais de $50 \%$ do total das árvores. Ligustrum lucidum W. T. Aiton representou 27,17\% do total de indivíduos arbóreos. Este valor percentual pode ser considerado alto, visto que Grey \& Deneke (1986) recomendam que uma única espécie não deva ultrapassar de 10 a $15 \%$ do total da população arbórea existente.

Uma porcentagem muito alta de uma mesma espécie poderá gerar riscos das plantas se tornarem alvo de pragas e doenças e conseqüentemente promoverem perdas significativas para o local. De acordo com Biondi \& Kischlat (2006), quanto maior o número de espécies presentes, maior será a capacidade de resistir às variações e de absorver impactos negativos, como a poluição e as adversidades climáticas, e menores serão as possibilidades do surgimento de pragas e doenças.

Quanto à procedência, $80,23 \%$ do total de indivíduos e $62,36 \%$ das espécies são exóticas. As espécies exóticas correspondem a 100\% das plantas herbáceas (Tabela 2), 78,38\% dos arbustos (Tabela 3), 75,00\% das palmeiras (Tabela 4) e $54,17 \%$ das árvores (Tabela 1 ). Conforme Biondi et al. (2008), a utilização de espécies exóticas diminui a chance dos estudantes conhecerem, no pátio das unidades de ensino, as espécies nativas do Brasil e do ecossistema local.

Em relação às espécies nativas, apenas $55,22 \%$ delas são ocorrentes no ecossistema Floresta Ombrófila Mista e podem ser consideradas ocorrentes na cidade de Curitiba (Tabela 1). Rizzo et al. (1993), em estudo das áreas verdes do Campus II da Universidade Federal de Goiás (UFG), verificaram que houve uma preferência pela introdução de espécies exóticas no Brasil e de nativas de outros ecossistemas, em detrimento das espécies da flora de Goiás. Isto se deu, provavelmente, em virtude da dificuldade de aquisição de mudas de espécies nativas locais quando da construção deste Campus. Lombardi \& Morais (2003), em levantamento das espécies cultivadas no Campus da Universidade Federal de Minas Gerais (UMG), encontraram apenas $47 \%$ das espécies nativas da flora local.

Um aspecto negativo associado a alta freqüência (mais de 25\%) de ocorrência do Ligustrum lucidum no Campus III - Centro Politécnico é que a espécie é considerada uma planta invasora pela Portaria IAP $n^{\circ} 095$ que reconhece a Lista Oficial de Espécies Exóticas Invasoras para o Estado do Paraná (PARANÁ, 2007). Originária da China e Coréia, segundo Lorenzi et al. (2003), ela é freqüente em parques e em arborização urbana e já foi considerada a "árvore ideal" para plantio em calçadas de ruas e avenidas. Introduzida voluntariamente para fins ornamentais, conforme Ziller et al. (2004), tornou-se invasora em ambientes urbanos e na Floresta Ombrófila Mista. Segundo Silva et al. (2007), a sua dispersão e seu crescimento ocorrem de maneira rápida fazendo-a competir e impedir a regeneração de plantas nativas.

Foram encontradas outras 10 espécies arbóreas reconhecidas como espécies exóticas invasoras em áreas de ocorrência da Floresta Ombrófila Mista no Estado do Paraná pela Portaria IAP $n^{\circ}$ 095/2007, sendo estas: Acacia mearnsii, Citrus limon, Eriobotrya japonica, Hovenia dulcis, Melia azedarach, Morus nigra, Pinus taeda, $P$. elliottii, Pittosporum undulatum e Psidium guajava (Tabela 1). Segundo esta portaria, os imóveis públicos nos quais for constatada a presença destas espécies exóticas invasoras devem obrigatoriamente proceder à sua erradicação ou controle para evitar a contaminação biológica (PARANÁ, 2007). De acordo com Blum et al. (2008), é de extrema importância que as exóticas invasoras sejam, de forma planejada e gradativa, substituídas por espécies preferencialmente nativas da região fitoecológica local. Estas devem, portanto, ser priorizadas nos planejamentos de manejo da arborização.

Os resultados acima mostram a presença de espécies inadequadas no Campus III - Centro Politécnico. Nobre \& Menezes (2006), em estudo da arborização do Campus da Universidade Federal do Amapá (UNIFAP), verificaram que a escolha das plantas nem sempre obedeceu a critérios de conservação ambiental, sendo práticas comuns o plantio e o manejo inadequado de espécies vegetais.

Quanto a existência de espécies com frutos consumidos por humanos, encontrou-se 22 espécies frutíferas arbóreas, representando 18,33\% das espécies e $21,12 \%$ dos indivíduos arbóreos (Tabela 1), uma espécie entre os arbustos (Tabela 2) e outra entre as herbáceas (Tabela 3). Observando-se as espécies com frutos consumidos pela avifauna, tem-se 39 espécies arbóreas (32,50\% das espécies e $51,62 \%$ dos indivíduos arbóreos), duas entre os arbustos, uma entre as plantas herbáceas e uma entre as palmeiras. Se realizado um plano de manejo para substituição das plantas invasoras, estes números devem diminuir, visto que muitas das espécies invasoras encontradas são frutíferas.

Os resultados indicam que não foi priorizado o uso de plantas frutíferas no Campus III - Centro Politécnico, uma vez que existe um ambiente com poucas relações com a fauna e até mesmo com a comunidade acadêmica. A presença de mais espécies frutíferas, além de produzir ambientes paisagisticamente atrativos aos usuários do Campus, poderá servir para aumentar a diversidade biológica local. Segundo Mendonça \& Anjos (2005), quanto maior a quantidade de árvores frutíferas no ambiente urbano, maior é a disponibilidade de micro ambientes ofertados à vida silvestre.

Destaca-se que no Campus III - Centro Politécnico há a presença de 157 exemplares de 
LEAL, L. et al. Censo da arborização do Campus III...

Araucaria angustifolia (Bertol.) Kuntze (pinheiro-doparaná), planta que deu origem ao nome do Estado do Paraná, e está na lista de plantas ameaçadas de extinção (BRASIL, 1992). Há ainda um exemplar de Caesalpinia echinata Lam. (pau-brasil), árvore símbolo do Brasil (BRASIL, 1978) e que deu origem ao nome do país. Eisenlohr et al. (2006) sugere o uso de plantas raras, como a Caesalpinia echinata, como uma forma de contribuir para sua preservação e para a prática de educação ambiental.

A arborização do Campus III - Centro Politécnico não seguiu nenhuma linha paisagística, seja conservacionista, estética ou funcional. Este diagnóstico da vegetação existente poderá servir de base a um plano diretor para uma arborização mais consistente, seguindo objetivos claros, eficientes e didáticos. Medidas de substituição de espécies inadequadas e plantio de espécies nativas deverão ser realizadas de modo a servir de exemplo para os estudantes, prefeituras e órgãos ambientais. Para que esse processo possa fazer parte das atividades pedagógicas e cotidianas de seus usuários será preciso criar ações que permitam o envolvimento da comunidade acadêmica.

\section{CONCLUSÕES}

Após o término desta pesquisa, pôde-se constatar que a vegetação que compõe a arborização do Campus do Centro Politécnico, introduzida pelos técnicos da Prefeitura Universitária, professores e estudantes, é predominantemente composta por espécies das famílias Fabaceae, Cupressaceae e Myrtaceae, pela forma de vida arbórea e de procedência exótica do Brasil. Embora o Campus apresente um grande número de plantas que podem ser usadas em aulas práticas e em pesquisas, encontram-se inadequada por apresentar um desequilíbrio entre 0 número de plantas por espécie, pouca representatividade de espécies nativas do ecossistema local e a presença de espécies invasoras.

Recomenda-se que sejam realizadas medidas de substituição das espécies invasoras e plantio de espécies nativas para adequação da arborização do Campus.

\section{REFERÊNCIAS}

1. BIONDI, D. Paisagismo. Recife: UFPE, $1990.180 \mathrm{p}$

2. BIONDI, D.; KISCHLAT, E. A vegetação urbana e a biodiversidade. Diálogo, n. 1, p.155-168, 2006.

3. BIONDI, D., LEAL, L; SCHAFFER, M. Aspectos importantes das plantas ornamentais em escolas públicas estaduais da cidade de Curitiba, PR. Revista Brasileira de Ciências Agrárias, v. 3, n. 3, p. 267-275, 2008.

4. BLUM, C. T.; BORGO, M.; SAMPAIO, A. C. F. Espécies exóticas invasoras na arborização de vias públicas de MaringáPR. Revista da Sociedade Brasileira de Arborização Urbana, v. 3, n. 2, p. 78-97, 2008.

5. BRASIL. Lei $\mathrm{n}^{0}$ 6.607, de 7 de dezembro de 1978. JusBrasil Legislação. 1978. Disponível em: <http:// www.jusbrasil.com.br/legislacao/128361/lei-6607-78>. Acesso em: 24 out. 2008.

6. BRASIL. Portaria IBAMA no 37- N, de 3 de abril de 1992. Legislação Ambiental. 1992. Disponível em: <http:// www.ipef.br/legislacao/bdlegislacao/detalhes.asp?ld=995>. Acesso em: 24 out. 2008.

7. EISENLOHR, P. V. et al. Espécies arbóreas empregadas na ornamentação do Campus-Sede da Universidade Federal de Viçosa, Viçosa, Minas Gerais, Brasil. In: CONGRESSO BRASILEIRO DE ARBORIZAÇÃO URBANA, 10., 2006, Maringá Anais... Maringá: SBAU, 2006.

8. FALEIRO, W.; AMÂNCIO-PEREIRA, F. Arborização viária do Campus Umuarama da Universidade Federal de Uberlândia, MG. Revista Científica Eletrônica de Engenharia Florestal, v. 6, n. 10, p. 1-17, 2007.

9. FEDRIZZI, B. Paisagismo no pátio escolar. Porto Alegre: UFRGS, 1999. 60 p.

10. FERRI, G. M. Botânica: morfologia externa das plantas [organografia]. 11. ed. São Paulo: Melhoramentos, 1976. 113 p.

11. GREY, G.W.; DENEKE, F.J. Urban Forestry. 2. ed. New York: John Wiley, 1986. 299 p.

12. KURIHARA, D. L.; IMAÑA-ENCIMAS, J.; PAULA, J. E. Levantamento da arborização do Campus da Universidade de Brasília. Cerne, v. 11, n. 2, p. 127-136, 2005.

13. LOMBARDI, J. A., MORAIS, P. O. Levantamento florístico das plantas empregadas na arborização do campus da Universidade Federal de Minas Gerais, Belo Horizonte-MG. Lundiana, v. 4, n. 2, p. 83-88, 2003.

14. LORENZI, H. Árvores brasileiras: manual de identificação e cultivo de plantas arbóreas nativas do Brasil. Nova Odessa: Plantarum, 1992. $368 \mathrm{p}$.

15. LORENZI, H. Árvores brasileiras: manual de identificação e cultivo de plantas arbóreas nativas do Brasil. Nova Odessa: Plantarum, 1998. v. 2. 376 p.

16. LORENZI, H.; SOUZA, H. M. Plantas ornamentais no Brasil: arbustivas, herbáceas e trepadeiras. 3. ed. Nova Odessa: Instituto Plantarum, 2001. $1088 \mathrm{p}$.

17. LORENZI, H. et al. Árvores exóticas no Brasil: madeireiras, ornamentais e aromáticas. Nova Odessa: Instituto Plantarum, 2003. 368 p.

18. MELO, E. F. R. Q.; SEVERO, B. M. A. Vegetação Arbórea do Campus da Universidade de Passo Fundo. Revista da Sociedade Brasileira de Arborização Urbana, v. 2, n. 2, p. 76-87, 2007.

19. MENDONÇA, L. B.; ANJOS, L. Beija-flores (aves, Trochilidae) e seus recursos florais em uma área urbana do Sul do Brasil. Revista Brasileira de Zoologia, v. 22, n. 1, p. 51-59, 2005.

20. MICHALENKO, G. A sustainable tree management vision for the University of Waterloo Campus: Greening the Campus Final Report. Waterloo: University of Waterloo, 2002. 44 p.

21. MISSOURI BOTANICAL GARDEN. Missouri Botanical Garden's VAST (Vascular Tropicos). 2008, Disponível em: <http://mobot.org/W3T/Search/vast.html>. Acesso em: 10 abril 2008.

22. NOBRE, F. R.; MENEZES, C. R. O Paisagismo das áreas de jardim do Campus Marco Zero do Equador da UNIFAP diagnóstico preliminar das espécies existentes. In: CONGRESSO NACIONAL DE BOTÂNICA, 57., 2006, Gramado. Resumos... Gramado: SBB, 2007. CD -ROM (Trabalho № 73) 
23. PAIVA, G. A. Levantamento florístico quali-quantitativo da Praça do Relógio da Cidade Universitária "Armando de Salles Oliveira" - USP/São Paulo. 2006. 90 f. Dissertação (Mestrado em Fitotecnia) - Escola Superior de Agricultura Luiz de Queiroz, Universidade de São Paulo. Piracicaba, 2006.

24. PAIVA, P. D. O. et al. Identificação e caracterização das espécies arbóreas do canteiro central da Universidade Federal de Lavras/MG. Ciência e Agrotecnologia, v. 28, n. 3, p. 515-519, 2004.

25. PARANÁ. Portaria IAP n 095, de 22 de maio de 2007. Reconhece a Lista Oficial de Espécies Exóticas Invasoras para o Estado do Paraná, estabelece normas de controle, revoga Portaria IAP n $^{\circ} 074 / 2007$ e dá outras providências. 2007. Disponível em: <http://www.iap.pr.gov.br/arquivos/File/iap/port_95_07.pdf>. Acesso em: 24 out. 2008.

26. PARMAKSIZ, A.; ATAMOV, V.; ASLAN, M. The flora of Osmanbey Campus of the Harran University. Journal of Biological Sciences, v. 6, n. 5, p. 793-804, 2006.

27. RIZZO, J. A.; RIBEIRO FILHO, J.; HASHIMOTO, M. Y. Estudo da arborizacão e das áreas verdes do Campus II da Universidade Federal de Goiás. Anais da Escola de Agronomia e Veterinária, v. 23, n. 1, p. 19-45, 1993.

28. SCHAFFER, M. Análise da qualidade ambiental dos terrenos das escolas estaduais do Município de Curitiba / PR. 2005. 201 f. Dissertação (Mestrado em Ciências do Solo) - Universidade Federal do Paraná, Curitiba, 2005.

29. SILVA, L. M. et al. Arborização de vias públicas e a utilização de espécies exóticas: o caso do bairro Centro de Pato Branco/PR. Scientia Agraria, v. 8, n. 1, p. 47-53, 2007.

30. ZILLER, S. R.; ZALBA, S. Propostas de ação para prevenção e controle de espécies exóticas invasoras. Natureza \& Conservação, v. 5, n. 2, p. 8-15, 2007.

31. ZILLER, S.R.; ZENNI, R. D.; GRAF NETO, J. Invasões biológicas: introdução, impactos e espécies invasoras no Brasil. In: PEDROSA-MACEDO, J. H.; BREDOW, E. A. (Ed.) Princípios e rudimentos do controle biológico de plantas: coletânea. Curitiba: UFPR. 2004. . p. 17-41.

Recebido em 16/12/2008 Aceito em 17/06/2009 
